\title{
Correction to: Comparative analysis of sperm motility in liquid and seminal coagulum portions between Bornean orangutan (Pongo pygmaeus) and chimpanzee (Pan troglodytes)
}

\author{
Kodzue Kinoshita $^{1}$ (D) Yoriko Indo ${ }^{2} \cdot$ Tomoyuki Tajima $^{3}$ (D) Noko Kuze ${ }^{4,5}$ (D) Etsuko Miyakawa ${ }^{6} \cdot$ Toshio Kobayashi $^{7}$. \\ Tomoyuki Nakamura $^{8} \cdot$ Mitsuaki Ogata $^{9} \cdot$ Fumihiko Okumura $^{10} \cdot$ Takashi Hayakawa $^{2,10,13}$ (D) Naruki Morimura ${ }^{1,11}$ (D) . \\ Yusuke Mori $^{1,11} \cdot$ Munehiro Okamoto $^{2} \cdot$ Yasuhiko Ozaki $^{12}$ (D) Satoshi Hirata ${ }^{1,11}$ (D)
}

Published online: 20 April 2021

(C) Japan Monkey Centre 2021

\section{Correction to: Primates https://doi.org/10.1007/s10329-020-00887-2}

In the original publication of the article, the coauthor "Takashi Hayakawa" was wrongly assigned as co-corresponding author.

The original article has been corrected.
The original article can be found online at https://doi.org/10.1007/ s10329-020-00887-2.

Kodzue Kinoshita

kinoshita.kodzue.8v@kyoto-u.ac.jp

$\triangle$ Satoshi Hirata

hirata.satoshi.8z@kyoto-u.ac.jp

1 Wildlife Research Center, Kyoto University, Kyoto, Kyoto 606-8203, Japan

2 Primate Research Institute, Kyoto University, Inuyama, Aichi 484-8506, Japan

3 Human Evolution Studies, Graduate School of Science, Kyoto University, Kyoto, Kyoto 606-8502, Japan

4 Japan Society for the Promotion of Science, Chiyoda, Tokyo 102-0083, Japan

5 The National Museum of Nature and Science, Tsukuba, Ibaraki 305-0005, Japan

6 Kanazawa Zoological Gardens, Yokohama, Kanagawa 236-0042, Japan
Publisher's Note Springer Nature remains neutral with regard to jurisdictional claims in published maps and institutional affiliations.
7 Zoorasia Yokohama Zoological Gardens, Yokohama, Kanagawa 241-0001, Japan

8 Chiba Zoological Park, Chiba, Chiba 264-0037, Japan

9 Preservation and Research Center, The City of Yokohama, Yokohama, Kanagawa 241-0001, Japan

10 Japan Monkey Centre, Inuyama, Aichi 484-0081, Japan

11 Kumamoto Sanctuary, Kyoto University, Uki, Kumamoto 869-3201, Japan

12 Department of Obstetrics and Gynecology, Graduate School of Medical Sciences, Nagoya City University, Nagoya, Aichi 467-8601, Japan

13 Present Address: Current address: Faculty of Environmental Earth Science, Hokkaido University, Sapporo, Hokkaido 060-0810, Japan 